\title{
EDITORIAL
}

\section{Innovation, or reverse causation?}

\author{
D. Rosen**\# and T. Frischer ${ }^{\star}$
}

L ongitudinal, multi-year cohort studies with large numbers of participants offer the only scientific possibility of clearly identifying the causes of childhood asthma (setting aside, for the moment, the difficulties in defining it). While the goal of these studies is clear, the exposure variables tested in them are often not well defined, and their main result is the generation of hypotheses which can then be tested in further studies. Limitations in funding, the enormous amount of time invested in the collection and subsequent analysis of data sets comprised of thousands of participants and several hundred variables, and the loss of patients to follow-up, all present formidable difficulties to investigators. Owing to their sheer size, these studies require the labour of numerous epidemiologists, post-doctoral fellows and bio-statisticians. Across their lifespan, some of the core understandings about the disease, the research questions and even statistical methods may undergo significant evolution and change. For example, the method of generalised estimating equations now used to analyse longitudinal data by regression analyses was only published in 1986 [1]. Because of all these reasons, one must recognise that the data structure of a study initiated in 1989 may not be appropriate for answering research questions posed 20 or $30 \mathrm{yrs}$ later. One also needs to be aware that the processing of large data sets through sophisticated statistical models enables researchers to find statistically significant correlations which can tempt them into formulating post hoc biological explanations for the unexpected relationships which emerge.

In this month's issue of the European Respiratory Journal, KOZYRSKYJ et al. [2] have published a paper summarising research looking at a possible relationship between parentally reported sleep fragmentation in children during their first 3 yrs of life and the subsequent development of asthma. The authors have concluded that there is indeed a correlation between sleep fragmentation during the first 3 yrs of life and the development of nonatopic asthma at ages 6 and 14 yrs.

Sleep fragmentation was assessed on a yearly basis at well-child visits at ages 1,2 and 3 yrs. Parents were asked to quantify the number of times their children had awakened weekly during the past year at these visits. Children who had awakened more than twice weekly were designated to have had fragmented sleep. Subsequently, at age 6 and $14 \mathrm{yrs}$, the children were assessed for the presence of physician-diagnosed asthma.

*Division of Respiratory Diseases, Children's Hospital Boston, and "Harvard Medical School, Boston, MA, USA. "Uni-Kinderklinik Wien, Vienna, Austria.

CORRESPONDENCE: D. Rosen, Division of Respiratory Diseases, Children's Hospital Boston, 300 Longwood Avenue, Boston, MA 02115, USA. E-mail: Dennis.Rosen@childrens.harvard.edu
Much information, however, is lacking, leading to serious questions about the significance of the findings and the validity of the authors' conclusions. There is no information given about the children's sleep patterns, the presence of underlying sleep or other medical disorders (including airway reactivity), or even who the caregiver providing the history was. Were the children described as having fragmented sleep being put in bed for more time than their age-appropriate sleep requirements, resulting in their sleep appearing to be fragmented, when in fact it was simply divided in a way which interfered with the parents' diurnal schedule? Was there a component of sleep association disorder, which can develop in, among others, nursing infants and toddlers who are allowed to fall asleep on the mother's breast? The researchers did find that children who were breastfed for $>6$ months had a statistically higher incidence of fragmented sleep. In that case, it would be very interesting to know whether breastfeeding had a protective or promoting effect upon the development of asthma. This was not mentioned, and is unfortunate, as breastfeeding has repeatedly been demonstrated to protect against the development of asthma $[3,4]$, and this could certainly have influenced the conclusions the authors reached.

Other questions include whether, in those infants who went on to be diagnosed with asthma, airway reactivity was present during the first 3 yrs of life, and whether that could have been the cause of their frequent awakenings, thereby constituting reverse causation, something frequently seen in asthma research [5]. One of the key problems of population-based asthma research is that exposure and outcome variables are very often tightly bound to each other, and disentangling the temporal relationships between cause and effect becomes exceedingly difficult. TURNER et al. [6] recently published findings showing that airway reactivity at $1 \mathrm{yr}$ of age correlates with increased asthma prevalence at age 11 yrs. While the authors did attempt to control for "wheeze during sleep" (table 3 in the article by KOZYRSKYJ et al. [2]), this was presumably parentally reported, and not a diagnosis made by a physician.

The methodology used to identify those children whose sleep was fragmented is also fraught with problems. Information is not given as to the identity of the parent filling out the survey. Depending upon the caregiving patterns of the specific families, there may have been significant discrepancies between how frequently the child was perceived to be awakening by the mother, herself waking up to nurse the child back to sleep, and by the father, who may have continued to sleep and remain oblivious to the events of the night. It also requires a great leap of faith to base one's conclusions upon a questionnaire asking parents to summarise their child's sleeping patterns over the 
preceding year without considering the possibility of significant recall bias. While the authors cite a study by SADEH [7] as proof that questionnaires can be used to accurately describe the sleep patterns of infants and toddlers, that particular study was based upon daily sleep logs which the parents kept during a 1-week period, which is much different from expecting parents to faithfully recapitulate the events of the previous 365 days. While the study carried out by SADEH [7] demonstrated agreement between sleep diaries and actigraphy in determining nocturnal sleep duration and nighttime awakenings in children aged 5-29 months, a different study by WERNER et al. [8] found sleep logs to be inferior to actigraphy in assessing nighttime awakenings in children aged 4-7 yrs, most likely because of the ability of many of these children to soothe themselves back to sleep without their parents being aware that they had awakened.

It is very important to point out that statistical analyses cannot overcome the fundamental problem of this study not having been designed a priori to investigate the effects of fragmented sleep on the subsequent development of asthma in children at a later age. The standard criteria to suggest a cause-effect relationship in epidemiological research include statistical significance, biological plausibility, analogous findings in other fields of medicine and repeated, similar observations in other populations, regions and/or time periods. Unfortunately, while the first criterion was been met by this study, the others were not.

While exciting as the underlying hypothesis is in proposing a novel and hitherto unexamined mechanism for the development of nonatopic asthma through the activation of the hypothalamic-pituitary axis by sleep fragmentation, it is very difficult to draw any meaningful conclusions from this particular study, because of the problems with its design.

\section{STATEMENT OF INTEREST}

None declared.

\section{REFERENCES}

1 Zeger SL, Liang KY. Longitudinal data analysis for discrete and continuous outcomes. Biometrics 1986; 42: 121-130.

2 Kozyrskyj AL, Kendall GE, Zubrick SR, et al. Frequent nocturnal awakening in early life is associated with nonatopic asthma in children. Eur Respir J 2009; 34: 1288-1295.

3 Scholtens S, Wijga AH, Brunekreef B, et al. Breast feeding, parental allergy and asthma in children followed for 8 years. The PIAMA birth cohort study. Thorax 2009; 64: 604-609.

4 Burr ML, Limb ES, Maguire MJ, et al. Infant feeding, wheezing, and allergy: a prospective study. Arch Dis Child 1993; 68: 724-728.

5 Frischer T, Kuehr J, Meinert R, et al. Maternal smoking in early childhood: a risk factor for bronchial responsiveness to exercise in primary-school children. J Pediatr 1992;121:17-22.

6 Turner SW, Young S, Goldblatt J, et al. Childhood asthma and increased airway responsiveness: a relationship that begins in infancy. Am J Respir Crit Care Med 2009; 179: 98-104.

7 Sadeh A. A brief screening questionnaire for infant sleep problems: validation and findings for an internet sample. Pediatrics 2004; 113: e570-e577.

8 Werner H, Luciano M, Guyer C, et al. Agreement rates between actigraphy, diary, and questionnaire for children's sleep patterns. Arch Pediatr Adolesc Med 2008; 162: 350-358. 